\title{
K3 SURFACES WITH AN ORDER 60 AUTOMORPHISM AND A CHARACTERIZATION OF SUPERSINGULAR K3 SURFACES WITH ARTIN INVARIANT 1
}

\author{
JONGHAE KEUM
}

\begin{abstract}
In characteristic $p=0$ or $p>5$, we show that a K3 surface with an order 60 automorphism is unique up to isomorphism. As a consequence, we characterize the supersingular K3 surface with Artin invariant 1 in characteristic $p \equiv 11(\bmod 12)$ by a cyclic symmetry of order 60 .
\end{abstract}

Let $X$ be a K3 surface over an algebraically closed field $k$ of characteristic $p \geq 0$. An automorphism $g$ of $X$ is called symplectic if it preserves a regular 2 -form $\omega_{X}$, and purely non-symplectic if no power of $g$ is symplectic except the identity.

Over $k=\mathbb{C}$, Xiao [17] and Machida and Oguiso [9] proved that a positive integer $N$ is the order of a purely non-symplectic automorphism of a complex K3 surface if and only if $\phi(N) \leq 20$ and $N \neq 60$, where $\phi$ is the Euler function. On the other hand, there is a K3 surface with an automorphism of order 60 ([7] Example 3.2):

$$
\begin{aligned}
X_{60}:= & \left(y^{2}+x^{3}+t_{0} t_{1}^{11}-t_{0}^{11} t_{1}=0\right) \subset \mathbb{P}(4,6,1,1), \\
& g_{60}\left(t_{0}, t_{1}, x, y\right)=\left(t_{0}, \zeta_{60}^{6} t_{1}, \zeta_{60}^{2} x, \zeta_{60}^{3} y\right),
\end{aligned}
$$

where $\zeta_{60} \in k$ is a primitive 60 th root of unity. The K3 surface $X_{60}$ is defined over the integers and both the surface and the automorphism have a good reduction mod $p$ unless $p=2,3,5$.

For an automorphism $g$ of finite order of a K3 surface $X$, we write

$$
\operatorname{ord}(g)=m \cdot n
$$

if $g$ is of order $m n$ and the natural homomorphism

$$
\langle g\rangle \rightarrow \operatorname{GL}\left(H^{0}\left(X, \Omega_{X}^{2}\right)\right)
$$

has kernel of order $m$ and image of order $n$. We call $n$ the non-symplectic order of $g$.

The main result of the paper is the following.

Theorem 0.1. Let $k$ be an algebraically closed field of characteristic $p=0$ or $p>5$. Let $X$ be a K3 surface defined over $k$ with an automorphism $g$ of order 60 . Then

(1) $\operatorname{ord}(g)=5.12$;

(2) the pair $(X,\langle g\rangle)$ is isomorphic to the pair $\left(X_{60},\left\langle g_{60}\right\rangle\right)$, i.e., there is an isomorphism $f: X \rightarrow X_{60}$ such that $f\langle g\rangle f^{-1}=\left\langle g_{60}\right\rangle$. 
The non-existence of a complex K3 surface with a purely non-symplectic automorphism of order 60 was proved by Machida and Oguiso [9]. Their proof does not extend to the positive characteristic case, as it uses the holomorphic Lefschetz formula and the notion of transcendental lattice, both not available in positive characteristic.

Theorem 0.1 and Main Theorem of [7] determine completely the list of all nonsymplectic orders in characteristic $p>0$ :

Corollary 0.2. In any fixed characteristic $p>0$, a positive integer $N$ is the nonsymplectic order of an automorphism of a K3 surface if and only if $p \nmid N, N \neq 60$ and $\phi(N) \leq 20$.

It is well known that the Fermat quartic surface

$$
x_{0}^{4}+x_{1}^{4}+x_{2}^{4}+x_{3}^{4}=0
$$

is a supersingular K3 surface with Artin invariant 1 , if the characteristic $p \equiv 3(\bmod$ 4). This can be seen by using the algorithm for determining the Artin invariant of a weighted Delsarte surface whose minimal resolution is a K3 surface $[4,15]$. The same algorithm shows that in characteristic $p \equiv 11(\bmod 12)$ the surface $X_{60}$ is a supersingular K3 surface with Artin invariant 1, hence is isomorphic to the Fermat quartic surface, since a supersingular K3 surface with Artin invariant 1 is unique up to isomorphism $[12,13]$.

Corollary 0.3. In characteristic $p \equiv 11(\bmod 12)$, the Fermat quartic surface is the only K3 surface with an order 60 automorphism.

Over $k=\mathbb{C}$, Oguiso [11] proved that the Fermat quartic surface is the only K3 surface with a faithful action of a nilpotent group of order $512=2^{9}$. Over $k=\mathbb{C}$, the surface $X_{60}$ is not isomorphic to the Fermat quartic surface, as the former admits a purely non-symplectic automorphism of order 12 , while the latter has Picard number 20, hence by Nikulin [10] does not admit a purely non-symplectic automorphism of order $n$ with $\phi(n)>2$.

Remark 0.4. In characteristic $p=11$ the Fermat quartic surface also admits a cyclic action of order 66 (Example 7.5 [7], also [8]) and a symplectic action of the simple groups $M_{22}, M_{11}$ and $L_{2}(11)$, where $M_{r}$ is one of the Mathieu groups [3].

Throughout this paper, whenever we work with $l$-adic cohomology we assume $l$ is any prime different from the characteristic.

\section{Notation}

- $\mathrm{NS}(X)$ : the Néron-Severi group of a variety $X$;

- $X^{g}=\operatorname{Fix}(g)$ : the fixed locus of an automorphism $g$ of $X$;

- $e(g):=e(\operatorname{Fix}(g))$, the Euler characteristic of $\operatorname{Fix}(g)$ for $g$ tame;

- $\operatorname{Tr}\left(g^{*} \mid H^{*}(X)\right):=\sum_{j=0}^{2 \operatorname{dim} X}(-1)^{j} \operatorname{Tr}\left(g^{*} \mid H_{\mathrm{et}}^{j}\left(X, \mathbb{Q}_{l}\right)\right)$.

For an automorphism $g$ of a K3 surface $X$,

- $\left[g^{*}\right]=\left[\lambda_{1}, \ldots, \lambda_{22}\right]$ : the list of the eigenvalues of $g^{*} \mid H_{\mathrm{et}}^{2}\left(X, \mathbb{Q}_{l}\right)$.

- $\zeta_{a}$ : a primitive ath root of unity in $\overline{\mathbb{Q}_{l}}$;

- $\left[\zeta_{a}: \phi(a)\right] \subset\left[g^{*}\right]$ : all primitive ath roots of unity appear in $\left[g^{*}\right]$ where $\phi$ is the Euler function and $\phi(a)$ indicates the number of conjugates. 
- $[\lambda . r] \subset\left[g^{*}\right]: \lambda$ repeats $r$ times in $\left[g^{*}\right]$.

- $\left[\left(\zeta_{a}: \phi(a)\right) . r\right] \subset\left[g^{*}\right]$ : the list $\zeta_{a}: \phi(a)$ repeats $r$ times in $\left[g^{*}\right]$.

\section{Preliminaries}

We first recall the following basic results from [7].

Proposition 1.1 ([6] 3.7.3). Let $g$ be an automorphism of a projective variety $X$ over an algebraically closed field $k$ of characteristic $p>0$. Let $l$ be a prime $\neq p$. Then the following hold true.

(1) The characteristic polynomial of $g^{*} \mid H_{\mathrm{et}}^{j}\left(X, \mathbb{Q}_{l}\right)$ has integer coefficients for each $j$. In particular, if for some positive integer $m$ a primitive $m$ th root of unity appears with multiplicity $r$ as an eigenvalue of $g^{*} \mid H_{\mathrm{et}}^{j}\left(X, \mathbb{Q}_{l}\right)$, then so does each of its conjugates.

(2) The characteristic polynomial of $g^{*}$ does not depend on the choice of cohomology, l-adic or crystalline.

Proposition 1.2 ([7] Proposition 2.1). Let $g$ be an automorphism of a projective variety $X$ over an algebraically closed field $k$ of characteristic $p>0$. Let $l$ be a prime $\neq p$. Then the following hold true.

(1) If $g$ is of finite order, then $g$ has an invariant ample divisor, and 1 is an eigenvalue of $g^{*} \mid H_{\mathrm{et}}^{2}\left(X, \mathbb{Q}_{l}\right)$.

(2) If $X$ is a K3 surface, $g$ is tame and $g^{*} \mid H^{0}\left(X, \Omega_{X}^{2}\right)$ has $\zeta_{n} \in k$ as an eigenvalue, then $g^{*} \mid H_{\mathrm{et}}^{2}\left(X, \mathbb{Q}_{l}\right)$ has $\zeta_{n} \in \overline{\mathbb{Q}_{l}}$ as an eigenvalue.

Recall that for a non-singular projective variety $Z$ in characteristic $p>0$, there is an exact sequence of $\mathbb{Q}_{l}$-vector spaces

$$
0 \rightarrow \mathrm{NS}(Z) \otimes \mathbb{Q}_{l} \rightarrow H_{\mathrm{et}}^{2}\left(Z, \mathbb{Q}_{l}\right) \rightarrow T_{l}^{2}(Z) \rightarrow 0,
$$

where $T_{l}^{2}(Z)=T_{l}(\operatorname{Br}(Z))$ in the standard notation in the theory of étale cohomology (see [14]). The Brauer group $\operatorname{Br}(Z)$ is known to be a birational invariant.

Proposition 1.3 ([7] Proposition 2.2). Let $Z$ be a non-singular projective variety in characteristic $p>0$. Let $g$ be an automorphism of $Z$ of finite order. Assume $l \neq p$. Then the following assertions are true.

(1) Both traces of $g^{*}$ on $\mathrm{NS}(Z)$ and on $T_{l}^{2}(Z)$ are integers.

(2) $\operatorname{rank} \mathrm{NS}(Z)^{g}=\operatorname{rank} \mathrm{NS}(Z /\langle g\rangle)$.

(3) $\operatorname{dim} H_{\mathrm{et}}^{2}\left(Z, \mathbb{Q}_{l}\right)^{g}=\operatorname{rank} \mathrm{NS}(Z)^{g}+\operatorname{dim} T_{l}^{2}(Z)^{g}$.

(4) If the minimal resolution $Y$ of $Z /\langle g\rangle$ has $T_{l}^{2}(Y)=0$ (this condition is satisfied if $Z /\langle g\rangle$ is rational or is birational to an Enriques surface), then

$$
\operatorname{dim} H_{\text {et }}^{2}\left(Z, \mathbb{Q}_{l}\right)^{g}=\operatorname{rank} \operatorname{NS}(Z)^{g} .
$$

Proposition 1.4 (Topological Lefschetz formula, cf. [1]). Let $X$ be a smooth projective variety over an algebraically closed field $k$ of characteristic $p>0$ and let $g$ be $a$ tame automorphism of $X$. Then $X^{g}=\operatorname{Fix}(g)$ is smooth and

$$
e(g):=e\left(X^{g}\right)=\operatorname{Tr}\left(g^{*} \mid H^{*}(X)\right) .
$$


A tame symplectic automorphism $h$ of a K3 surface has finitely many fixed points, the number of fixed points $f(h)$ depends only on the order of $h$ and the list of possible pairs $(\operatorname{ord}(h), f(h))$ is the same as in the complex case ([2] Theorem 3.3 and Proposition 4.1). See also Nikulin's paper [10] for the complex case:

$$
(\operatorname{ord}(h), f(h))=(2,8),(3,6),(4,4),(5,4),(6,2),(7,3),(8,2) .
$$

Lemma 1.5 ([7] Lemma 2.6). Let $h$ be a tame symplectic automorphism of a K3 surface $X$. Then $h^{*} \mid H_{\mathrm{et}}^{2}\left(X, \mathbb{Q}_{l}\right)$ has eigenvalues

$$
\begin{array}{llll}
\operatorname{ord}(h)=2 & : & {\left[h^{*}\right]=[1,1.13,-1.8]} \\
\operatorname{ord}(h)=3 & : & {\left[h^{*}\right]=\left[1,1.9,\left(\zeta_{3}: 2\right) .6\right]} \\
\operatorname{ord}(h)=4 & : & {\left[h^{*}\right]=\left[1,1.7,\left(\zeta_{4}: 2\right) .4,-1.6\right]} \\
\operatorname{ord}(h)=5 & : & {\left[h^{*}\right]=\left[1,1.5,\left(\zeta_{5}: 4\right) .4\right]} \\
\operatorname{ord}(h)=6 & : & {\left[h^{*}\right]=\left[1,1.5,\left(\zeta_{3}: 2\right) .4,\left(\zeta_{6}: 2\right) .2,-1.4\right]} \\
\operatorname{ord}(h)=7 & : & {\left[h^{*}\right]=\left[1,1.3,\left(\zeta_{7}: 6\right) .3\right]} \\
\operatorname{ord}(h)=8 & : & {\left[h^{*}\right]=\left[1,1.3,\left(\zeta_{8}: 4\right) .2,\left(\zeta_{4}: 2\right) .3,-1.4\right]}
\end{array}
$$

where the first eigenvalue corresponds to an invariant ample divisor.

We need the following information on a special involution of a K3 surface.

Lemma 1.6. Let $X$ be a K3 surface in characteristic $p \neq 2$. Assume that $h$ is an automorphism of order 2 with $\operatorname{dim} H_{\mathrm{et}}^{2}\left(X, \mathbb{Q}_{l}\right)^{h}=2$. Then $h$ is non-symplectic and has an h-invariant elliptic fibration $\psi: X \rightarrow \mathbf{P}^{1}$,

$$
X /\langle h\rangle \cong \mathbf{F}_{e}
$$

a rational ruled surface, and $X^{h}$ is either a curve of genus 9 which is a 4-section of $\psi$ or the union of a section and a curve of genus 10 which is a 3-section. In the first case $e=0,1$ or 2 , and in the second $e=4$. Each singular fibre of $\psi$ is of type $I_{1}$ (nodal), $I_{2}, I I$ (cuspidal) or III, and is intersected by $X^{h}$ at the node and two smooth points if of type $I_{1}$, at the two singular points if of type $I_{2}$, at the cusp with multiplicity 3 and a smooth point if of type II, at the singular point tangentially to both components if of type III. If $X^{h}$ contains a section, then each singular fibre is of type $I_{1}$ or II.

Proof. Since $\operatorname{dim} H_{\mathrm{et}}^{2}\left(X, \mathbb{Q}_{l}\right)^{h}=2$, the eigenvalues of $h^{*} \mid H_{\mathrm{et}}^{2}\left(X, \mathbb{Q}_{l}\right)$ must be

$$
\left[h^{*}\right]=[1.2,-1.20], \text { so } \operatorname{Tr}\left(h^{*} \mid H^{*}(X)\right)=-16 .
$$

By Lemma 1.5, $h$ is non-symplectic, thus $X^{h}$ is a disjoint union of smooth curves and the quotient $X /\langle h\rangle$ is a non-singular rational surface. By Proposition 1.3, $X /\langle h\rangle$ has Picard number 2, hence is isomorphic to a rational ruled surface $\mathbf{F}_{e}$. Note that $e\left(X^{h}\right)=-16$, so $X^{h}$ is non-empty and has at most two components. Thus $X^{h}$ is either a curve $C_{9}$ of genus 9 or the union of two curves $C_{0}$ and $C_{10}$ of genus 0 and 10 , respectively. In the first case, the image $C_{9}^{\prime} \subset \mathbf{F}_{e}$ of $C_{9}$ satisfies $C_{9}^{\prime 2}=32$ and $C_{9}^{\prime} K=-16$, hence $C_{9}^{\prime} \equiv 4 S_{0}+(4+2 e) F$, where $S_{0}$ is the section with $S_{0}^{2}=-e$, and $F$ a fibre of $\mathbf{F}_{e}$. Since $S_{0} C_{9}^{\prime} \geq 0$, we have $e \leq 2$. In the second case, the image $C_{0}^{\prime}$ of $C_{0}$ has $C_{0}^{\prime 2}=-4$, hence $C_{0}^{\prime}=S_{0}$ and $e=4$, then it is easy to see that $C_{10}^{\prime} \equiv 3\left(S_{0}+4 F\right)$.

In characteristic $p \neq 3$ the pull-back of the ruling on $\mathbf{F}_{e}$ gives an $h$-invariant elliptic fibration $\psi: X \rightarrow \mathbf{P}^{1}$. Each singular fibre has at most two components since it is the pull-back of a fibre of $\mathbf{F}_{e}$. 
In characteristic $p=3$, we have to show that the pull-back is not a quasi-elliptic fibration. Suppose it is. The closure of the cusps of irreducible fibres is a smooth rational curve and must be fixed pointwise by $h$, then the genus 10 curve must be a section of the quasi-elliptic fibration, impossible.

The following easy lemmas also will be used frequently.

Lemma 1.7 ([7] Lemma 2.10). Let $S$ be a set and $\operatorname{Aut}(S)$ be the group of bijections of $S$. For any $g \in \operatorname{Aut}(S)$ and positive integers $a$ and $b$,

(1) $\operatorname{Fix}(g) \subset \operatorname{Fix}\left(g^{a}\right)$;

(2) $\operatorname{Fix}\left(g^{a}\right) \cap \operatorname{Fix}\left(g^{b}\right)=\operatorname{Fix}\left(g^{d}\right)$ where $d=\operatorname{gcd}(a, b)$;

(3) $\operatorname{Fix}(g)=\operatorname{Fix}\left(g^{a}\right)$ if $\operatorname{ord}(g)$ is finite and prime to $a$.

Lemma 1.8 ([7] Lemma 2.11). Let $R(n)$ be the sum of all primitive $n$th root of unity in $\overline{\mathbb{Q}}$ or in $\overline{\mathbb{Q}}$. Then

$$
R(n)=\left\{\begin{array}{ccl}
0 & \text { if } & n \text { has a square factor, } \\
(-1)^{t} & \text { if } \quad n \text { is a product of } t \text { distinct primes. }
\end{array}\right.
$$

The following lemma will play a key role in our proof.

Lemma 1.9. Let $g$ be an automorphism of order 60 of a K3 surface in characteristic $p \neq 2,3,5$. If

$$
\left[g^{*}\right]=\left[1, \zeta_{60}: 16, \zeta_{12}: 4, \pm 1\right]
$$

then

(1) There is a g-invariant elliptic fibration $\psi: X \rightarrow \mathbf{P}^{1}$ with 12 cuspidal fibres, say $F_{\infty}, F_{0}, F_{t_{1}}, \ldots, F_{t_{10}}$

(2) $\operatorname{Fix}\left(g^{30}\right)$ consists of a section $R$ of $\psi$ and a curve $C_{10}$ of genus 10 which is a 3 -section passing through each cusp with multiplicity 3 ;

(3) The action of $g$ on the base $\mathbf{P}^{1}$ is of order 10 , fixing 2 points, say $\infty$ and 0 , and makes the 10 points $t_{1}, \ldots, t_{10}$ to form a single orbit;

(4) $\operatorname{Fix}\left(g^{10}\right)=R \cup\{$ the cusps of the 12 cuspidal fibres $\}$;

(5) $\operatorname{Fix}\left(g^{12}\right)=\operatorname{Fix}(g)$ and it consists of the 4 points,

$$
R \cap F_{\infty}, \quad R \cap F_{0}, \quad C_{10} \cap F_{\infty}, \quad C_{10} \cap F_{0} ;
$$

(6) $\left[g^{*}\right]=\left[1, \zeta_{60}: 16, \zeta_{12}: 4,1\right]$.

Proof. Note that $\left[g^{30 *}\right]=[1,-1.16,-1.4,1]$, and

$$
\left[g^{10 *}\right]=\left[1,\left(\zeta_{6}: 2\right) .8,\left(\zeta_{6}: 2\right) .2,1\right], \quad e\left(g^{10}\right)=14 .
$$

Thus, we can apply Lemma 1.6 to $h=g^{30}$. Since

$$
\operatorname{Fix}\left(g^{d}\right) \subset \operatorname{Fix}\left(g^{30}\right)
$$

for any $d$ dividing 30, we see that $\operatorname{Fix}\left(g^{10}\right)$ consists of 14 points if $\operatorname{Fix}\left(g^{30}\right)$ is irreducible. If $\operatorname{Fix}\left(g^{30}\right)$ is a curve $C_{9}$ of genus 9 , then $g^{10}$ acts on $C_{9}$ with 14 fixed points, too many for an order 3 automorphism $g^{10} \mid C_{9}$. In fact, $e\left(g^{10} \mid C_{9}\right) \leq 11$ by the topological Lefschetz formula (Proposition 1.4) for $g^{10} \mid C_{9}$. This type of argument will frequently appear in the rest. Thus Fix $\left(g^{30}\right)$ consists of a section $R$ of a $g^{30}$-invariant elliptic fibration

$$
\psi: X \rightarrow \mathbf{P}^{1}
$$


and a curve $C_{10}$ of genus 10 which is a 3 -section. We know that

$$
X /\left\langle g^{30}\right\rangle \cong \mathbf{F}_{4}
$$

a rational ruled surface. Every automorphism of $\mathbf{F}_{4}$, hence the one induced by $g$, preserves the unique ruling, so $g$ preserves the elliptic fibration. Let $a$ and $b$ be the number of singular fibres of type $I_{1}$ and $I I$, respectively. Then

$$
a+2 b=e(X)=24, \quad 12 \leq a+b \leq 24 .
$$

Note that $g^{30}$ acts trivially on the base $\mathbf{P}^{1}$. Neither $g^{5}$ nor $g^{6}$ acts trivially on $\mathbf{P}^{1}$. Otherwise, $\operatorname{Fix}\left(g^{5}\right)$ or $\operatorname{Fix}\left(g^{6}\right)$ must contain the section $R$, the nodes of the nodal fibres and the cusps of the cuspidal fibres, too many, as we compute $e\left(g^{5}\right)=3 \pm 1$ and $e\left(g^{6}\right)=4$. Our automorphism $g$ acts on the set of the base points of the $a+b$ singular fibres. An orbit of this action has length 1, 2, 3, 5, 6, 10 or 15, i.e., a divisor of 30. If an orbit has length 3,5 or 6 , then $g^{5}$ or $g^{6}$ fixes all points in the orbit, hence acts trivially on the base $\mathbf{P}^{1}$. Thus no orbit has length $3,5,6$. If an orbit has length 15 , then $a \geq 16$ and $g^{2}$ fixes more than two points on the base $\mathbf{P}^{1}$. We have proved that every orbit has length 1,2 or 10 . Then $g^{10}$ fixes all base points of the singular fibres. Thus it acts trivially on the base $\mathbf{P}^{1}$ and $\operatorname{Fix}\left(g^{10}\right)$ contains $R$ and the nodes and the cusps of the singular fibres. Since $e\left(g^{10}\right)=14$, we infer that $a=0$ and $b=12$. Then the action of $g$ on the 12 base points of the cuspidal fibres has an orbit of length 10 ; otherwise $g^{2}$ would act trivially on the base. If the remaining two points, say $\infty$ and 0 , are interchanged by $g$, then $g$ fixes 2 points on the base $\mathbf{P}^{1}$ away from the 12 points, then $g^{2}$ fixes 4 points on the base, so acts trivially on the base. Thus $g$ fixes $\infty$ and 0 . This proves (1), (2), (3) and (4).

By (3) $\operatorname{Fix}(g)$ consists of the 4 points, hence $e(g)=\operatorname{Tr}\left(g^{*} \mid H^{*}(X)\right)=4$. Again, by (3) $\operatorname{Fix}\left(g^{12}\right)$ is a subset of $F_{\infty} \cup F_{0}$. Since $e\left(g^{12}\right)=4, \operatorname{Fix}\left(g^{12}\right)$ cannot contain any point other than the 4 points of $\operatorname{Fix}(g)$. This proves (5) and (6).

\section{Proof: the tame case}

Throughout this section, we assume that the characteristic $p>0, p \neq 2,3,5$ and $g$ is an automorphism of order 60 of a K3 surface. We first prove that $g$ cannot be purely non-symplectic.

Lemma 2.1. $\operatorname{ord}(g) \neq 1.60$.

Proof. Suppose that $\operatorname{ord}(g)=1.60$. Then by Proposition 1.2 the action of $g^{*}$ on $H_{\mathrm{et}}^{2}\left(X, \mathbb{Q}_{l}\right), l \neq \operatorname{char}(k)$, has $\zeta_{60} \in \overline{\mathbb{Q}_{l}}$ as an eigenvalue and

$$
\left[g^{*}\right]=\left[1, \zeta_{60}: 16, \eta_{1}, \ldots, \eta_{5}\right]
$$

where $\left[\eta_{1}, \ldots, \eta_{5}\right]$ is a combination of $\zeta_{12}: 4, \zeta_{10}: 4, \zeta_{5}: 4, \zeta_{6}: 2, \zeta_{4}: 2, \zeta_{3}: 2, \pm 1$, and the first eigenvalue corresponds to a $g$-invariant ample divisor.

Claim 1: $\left[\eta_{1}, \ldots, \eta_{5}\right] \neq\left[\zeta_{10}: 4, \pm 1\right],\left[\zeta_{5}: 4, \pm 1\right]$.

Suppose that $\left[\eta_{1}, \ldots, \eta_{5}\right]=\left[\zeta_{10}: 4, \pm 1\right]$ or $\left[\zeta_{5}: 4, \pm 1\right]$. Then Lefschetz fixed point formula gives

$$
e\left(g^{30}\right)=\operatorname{Tr}\left(g^{30 *} \mid H^{*}(X)\right)=-8
$$

and $\operatorname{Fix}\left(g^{30}\right)$ consists of $d$ smooth rational curves and a curve $C_{d+5}$ of genus $d+5$. We have $0 \leq d \leq 5$, since each fixed curve gives an invariant vector in $\operatorname{dim} H_{\text {et }}^{2}\left(X, \mathbb{Q}_{l}\right)$. 
Note that $e\left(g^{2}\right)=\operatorname{Tr}\left(g^{2 *} \mid H^{*}(X)\right)=1$. Since $\operatorname{Fix}\left(g^{2}\right) \subset \operatorname{Fix}\left(g^{30}\right)$, we infer that $\operatorname{Fix}\left(g^{2}\right)$ consists of a point. Note that $C_{d+5} \nsubseteq \mathrm{Fix}\left(g^{10}\right)$, since $e\left(g^{10}\right)=16>e\left(g^{30}\right)$. If $d=1,2$ or 4 , then $g$ acts on the $d$ smooth rational curves and $g^{2}$ preserves at least one of them, hence fixes at least 2 points. If $d=3$, then $g$ must rotate the 3 smooth rational curves and $g^{10}$ acts on the curve $C_{8}$ with 16 fixed points, which is impossible. If $d=0$, then $g^{10}$ gives an order 3 automorphism of the curve $C_{5}$ with 16 fixed points, impossible. If $d=5$, then $g$ must rotate the 5 smooth rational curves and $g^{5}$ preserves each of them, hence $e\left(g^{5}\right) \geq 10$. But $\operatorname{Tr}\left(g^{5 *} \mid H^{*}(X)\right) \leq 8$, contradicting the Lefschetz fixed point formula.

Claim 2: $\left[\eta_{1}, \ldots, \eta_{5}\right] \neq\left[\zeta_{6}: 2, \pm 1, \pm 1, \pm 1\right],\left[\zeta_{3}: 2, \pm 1, \pm 1, \pm 1\right]$.

Suppose that $\left[\eta_{1}, \ldots, \eta_{5}\right]=\left[\zeta_{6}: 2, \pm 1, \pm 1, \pm 1\right]$ or $\left[\zeta_{3}: 2, \pm 1, \pm 1, \pm 1\right]$. This case can be handled similarly. We see that $e\left(g^{30}\right)=-8$ and Fix $\left(g^{30}\right)$ consists of $d$ smooth rational curves and a curve $C_{d+5}$ of genus $d+5,0 \leq d \leq 5$. We also see that $e\left(g^{2}\right)=3$ and $\operatorname{Fix}\left(g^{2}\right)$ consists of either 3 points or a point and a $\mathbb{P}^{1}$. Note that $C_{d+5} \nsubseteq \operatorname{Fix}\left(g^{10}\right)$, since $e\left(g^{10}\right)=13>e\left(g^{30}\right)$. If $d=0$ or 1 , then $g^{10}$ gives an order 3 automorphism of the curve $C_{d+5}$ with at least 11 fixed points, which is impossible. If $d=2$, then $g^{2}$ preserves 2 smooth rational curves, hence fixes at least 4 points. If $d=3$, then $g$ must rotate the 3 smooth rational curves and $g^{10}$ acts on the curve $C_{8}$ with 13 fixed points, impossible. If $d=4$, then $g^{3}$ preserves each of them, hence $e\left(g^{3}\right) \geq 8$ or $e\left(g^{3}\right)=8+e\left(C_{9}\right)=-8$, which is possible only if $\left[g^{*}\right]=\left[1, \zeta_{60}: 16, \zeta_{3}: 2,1,1,1\right]$. Then $e(g)=5>e\left(g^{2}\right)$, but $\operatorname{Fix}(g)$ and $\operatorname{Fix}\left(g^{2}\right)$ consist of isolated points and some $\mathbb{P}^{1}$ 's. If $d=5$, then $g$ must rotate the 5 smooth rational curves and $g^{5}$ preserves each of them, hence $e\left(g^{5}\right) \geq 10$. But $\operatorname{Tr}\left(g^{5 *} \mid H^{*}(X)\right) \leq 7$, contradicting the Lefschetz formula.

Claim 3: $\left[\eta_{1}, \ldots, \eta_{5}\right] \neq\left[\left(\zeta_{6}: 2\right) .2, \pm 1\right],\left[\left(\zeta_{3}: 2\right) .2, \pm 1\right],\left[\zeta_{6}: 2, \zeta_{3}: 2, \pm 1\right]$.

Suppose that $\left[\eta_{1}, \ldots, \eta_{5}\right]=\left[\left(\zeta_{6}: 2\right) .2, \pm 1\right],\left[\left(\zeta_{3}: 2\right) .2, \pm 1\right]$ or $\left[\zeta_{6}: 2, \zeta_{3}: 2, \pm 1\right]$. Note that $e\left(g^{30}\right)=-8$ and $\operatorname{Fix}\left(g^{30}\right)$ consists of $d$ smooth rational curves and a curve $C_{d+5}$ of genus $d+5,0 \leq d \leq 5$. We see that $e\left(g^{2}\right)=0$. Since $\operatorname{Fix}\left(g^{2}\right) \subseteq \operatorname{Fix}\left(g^{30}\right), \operatorname{Fix}\left(g^{2}\right)=\emptyset$, thus $\operatorname{Fix}(g)=\emptyset$ and $\left[g^{*}\right]=\left[1, \zeta_{60}: 16,\left(\zeta_{3}: 2\right) .2,-1\right]$. Note that $C_{d+5} \nsubseteq \operatorname{Fix}\left(g^{10}\right)$, since $e\left(g^{10}\right)=10>e\left(g^{30}\right)$. If $d=0$, then $g^{10}$ gives an order 3 automorphism of the curve $C_{5}$ with 10 fixed points, which is impossible. If $d=1,2$ or 4 , then $g^{2}$ preserves at least one smooth rational curve, hence fixes at least 2 points. If $d=3$, then $g$ must rotate the 3 smooth rational curves, hence $g^{15}$ acts freely on the curve $C_{8}$, since $e\left(g^{15}\right)=6$. But no genus 8 curve admits a free involution. If $d=5$, then $g$ must rotate the 5 smooth rational curves and $g^{5}$ preserves each of them, hence $e\left(g^{5}\right) \geq 10$. But $\operatorname{Tr}\left(g^{5 *} \mid H^{*}(X)\right)=0$.

Claim 4: $\left[\eta_{1}, \ldots, \eta_{5}\right] \neq\left[\zeta_{4}: 2, \zeta_{6}: 2, \pm 1\right],\left[\zeta_{4}: 2, \zeta_{3}: 2, \pm 1\right]$.

Suppose that $\left[\eta_{1}, \ldots, \eta_{5}\right]=\left[\zeta_{4}: 2, \zeta_{6}: 2, \pm 1\right]$ or $\left[\zeta_{4}: 2, \zeta_{3}: 2, \pm 1\right]$. In this case, $e\left(g^{30}\right)=-12$ and $\operatorname{Fix}\left(g^{30}\right)$ consists of $d$ smooth rational curves and a curve $C_{d+7}$ of genus $d+7,0 \leq d \leq 3$. We compute

$$
e\left(g^{2}\right)=\operatorname{Tr}\left(g^{2 *} \mid H^{*}(X)\right)=-1>e\left(g^{30}\right),
$$

hence $C_{d+7} \nsubseteq \operatorname{Fix}\left(g^{2}\right)$. But then $e\left(g^{2}\right) \geq 0$. 
Claim 5: $\left[\eta_{1}, \ldots, \eta_{5}\right] \neq\left[\left(\zeta_{4}: 2\right) .2, \pm 1\right]$.

Suppose that $\left[\eta_{1}, \ldots, \eta_{5}\right]=\left[\left(\zeta_{4}: 2\right) .2, \pm 1\right]$. In this case, $e\left(g^{30}\right)=-16$ and $\operatorname{Fix}\left(g^{30}\right)$ consists of $d$ smooth rational curves and a curve $C_{d+9}$ of genus $d+9,0 \leq d \leq 1$. Since $e\left(g^{2}\right)=-2>e\left(g^{30}\right), C_{d+9} \nsubseteq \operatorname{Fix}\left(g^{2}\right)$, but then $e\left(g^{2}\right) \geq 0$.

Claim 6: $\left[\eta_{1}, \ldots, \eta_{5}\right] \neq\left[\zeta_{4}: 2, \pm 1, \pm 1, \pm 1\right]$.

Suppose that $\left[\eta_{1}, \ldots, \eta_{5}\right]=\left[\zeta_{4}: 2, \pm 1, \pm 1, \pm 1\right]$. In this case, $e\left(g^{30}\right)=-12$ and $\operatorname{Fix}\left(g^{30}\right)$ consists of $d$ smooth rational curves and a curve $C_{d+7}$ of genus $d+7,0 \leq$ $d \leq 3$. We compute

$$
e\left(g^{2}\right)=\operatorname{Tr}\left(g^{2 *} \mid H^{*}(X)\right)=2>e\left(g^{30}\right),
$$

hence $C_{d+7} \nsubseteq \operatorname{Fix}\left(g^{2}\right)$ and $\operatorname{Fix}\left(g^{2}\right)$ consists of either 2 points or a $\mathbb{P}^{1}$, since $\operatorname{Fix}\left(g^{2}\right) \subset$ $\operatorname{Fix}\left(g^{30}\right)$. Since $\operatorname{Fix}(g) \subset \operatorname{Fix}\left(g^{2}\right)$, we infer that

$$
e(g)=2 \text { or } 0 .
$$

By computing $\left[g^{15 *}\right]$ and $\left[g^{10 *}\right]$, we see that

$$
e(g)=e\left(g^{15}\right) \text { and } e\left(g^{10}\right)=12 .
$$

If $d=0$, then $g^{10}$ gives an order 3 automorphism of the curve $C_{7}$ with 12 fixed points, impossible. If $d=2$, then $g^{2}$ preserves both smooth rational curves, hence $e\left(g^{2}\right) \geq 4$. If $d=3$, then $g^{2}$ cannot preserve two of the three smooth rational curves, hence $g$ must rotate the three, then $g^{15}$ preserves each of the three, hence $e\left(g^{15}\right) \geq 6$. If $d=1$, then $g^{15}$ acts freely on the curve $C_{8}$. But no genus 8 curve admits a free involution.

Claim \%: $\left[\eta_{1}, \ldots, \eta_{5}\right] \neq[ \pm 1, \pm 1, \pm 1, \pm 1, \pm 1]$.

Suppose that $\left[\eta_{1}, \ldots, \eta_{5}\right]=[ \pm 1, \pm 1, \pm 1, \pm 1, \pm 1]$. In this case,

$e\left(g^{30}\right)=-8$ and $\operatorname{Fix}\left(g^{30}\right)$ consists of $d$ smooth rational curves and a curve $C_{d+5}$ of genus $d+5,0 \leq d \leq 5$. We also compute

$$
e\left(g^{2}\right)=6, \quad e\left(g^{15}\right)=e(g), \quad e\left(g^{10}\right)=16 .
$$

Since $e\left(g^{2}\right)>e\left(g^{30}\right)$, we see that $C_{d+5} \nsubseteq \operatorname{Fix}\left(g^{2}\right)$ and

$$
e\left(g^{15}\right)=e(g) \leq e\left(g^{2}\right)=6 .
$$

If $d \leq 2$, then $g^{10}$ gives an order 3 automorphism of the curve $C_{d+5}$ with $16-2 d$ fixed points, which is impossible. Assume $d \geq 4$. If $g^{15}$ preserves at least 4 of the $d$ smooth rational curves, then $e\left(g^{15}\right) \geq 8>6$. If $g^{15}$ preserves at most 2 of the $d$ smooth rational curves, then $g^{2}$ preserves at least 4 , hence $e\left(g^{2}\right) \geq 8>6$. If $g^{15}$ preserves exactly 3 of the $d$ smooth rational curves, then $d=5$ and $g^{15}$ acts freely as an involution on the curve $C_{10}$, a contradiction. Assume $d=3$. If $g$ rotates the 3 smooth rational curves or fixes each of them, then $g^{15}$ fixes each of them, hence acts freely on the curve $C_{8}$, a contradiction. If $g$ fixes exactly one of the 3 smooth rational curves, then $g^{2}$ fixes each of them, hence acts freely on the curve $C_{8}$, then $g$ acts freely on the curve $C_{8}$ and $e(g)=2$, then $g^{15}$ has $e\left(g^{15}\right)=2$, hence acts freely on the curve $C_{8}$. This proves the claim.

We may assume that $\left[g^{*}\right]=\left[1, \zeta_{60}: 16, \zeta_{12}: 4, \pm 1\right]$. Then by Lemma 1.9

$$
\left[g^{*}\right]=\left[1, \zeta_{60}: 16, \zeta_{12}: 4,1\right] \text {. }
$$


Consider the order 5 automorphism $g^{12}$. It is non-symplectic and the quotient

$$
X^{\prime}:=X /\left\langle g^{12}\right\rangle
$$

is a singular rational surface with $K_{X^{\prime}}$ numerically trivial. Furthermore, by Proposition 1.3 Picard number $\rho\left(X^{\prime}\right)=6$.

Claim 8: $X^{\prime}=X /\left\langle g^{12}\right\rangle$ has four singular points, one of type $\frac{1}{5}(3,3)$ and three of type $\frac{1}{5}(2,4)$.

To prove the claim, note first that $\operatorname{Fix}\left(g^{12}\right)$ consists of the 4 points from Lemma 1.9, 2 points of $R$ and 2 points of $C_{10}$. Since

$$
g^{12 *} \omega_{X}=\zeta_{5} \omega_{X} \text { for some } \zeta_{5} \in k,
$$

there are two types of local action of $g^{12}$ at a fixed point, $\frac{1}{5}(3,3)$ and $\frac{1}{5}(2,4)$. Let $a$ and $b$ be the number of points respectively of the two types. Then

$$
a+b=4 \text {. }
$$

Let $\varepsilon: Y \rightarrow X^{\prime}$ be the minimal resolution. Then

$$
K_{Y}=\varepsilon^{*} K_{X^{\prime}}-\sum D_{p}
$$

where $D_{p}$ is an effective $\mathbb{Q}$-divisor supported on the exceptional set of the singular point $p \in X^{\prime}$. Here "=" means numerical equivalence. Thus

$$
K_{Y}^{2}=\sum D_{p}^{2}=-\sum K_{Y} D_{p}
$$

See, e.g., Lemma 3.6 [5] for the formulas of $D_{p}$ and $K_{Y} D_{p}$, which are valid not only in the complex case but also for tame quotient singular points in positive characteristic.

We compute

$$
K_{Y}^{2}=10-\rho(Y)=10-\left\{\rho\left(X^{\prime}\right)+a+2 b\right\}=4-a-2 b .
$$

On the other hand, $K_{Y} D_{p}=\frac{9}{5}$ if $p$ is of type $\frac{1}{5}(3,3)$, and $K_{Y} D_{p}=\frac{2}{5}$ if $p$ is of type $\frac{1}{5}(2,4)$, thus

$$
K_{Y}^{2}=-\frac{9}{5} a-\frac{2}{5} b
$$

Solving the system, we get $a=1$ and $b=3$. This proves the claim.

Now by Claim 8, we compute that

$$
K_{Y}=-\frac{3 A}{5}-\sum_{i=1}^{3} \frac{A_{1 i}+2 A_{2 i}}{5}
$$

where $A$ and $A_{j i}$ are exceptional curves with $A^{2}=-5, A_{1 i}^{2}=-2, A_{2 i}^{2}=-3, A_{1 i} \cdot A_{2 i}=$ 1. If the 2 points of $R$ are of type $\frac{1}{5}(2,4)$, then the proper transform $R^{\prime}$ of the image of $R$ in $X^{\prime}$ has intersection number with $K_{Y}$,

$$
K_{Y} \cdot R^{\prime}=-\frac{1}{5}-\frac{1}{5},-\frac{1}{5}-\frac{2}{5} \text { or }-\frac{2}{5}-\frac{2}{5},
$$

none is an integer. If the 2 points of $C_{10}$ are of type $\frac{1}{5}(2,4)$, then the proper transform $C_{10}^{\prime}$ of the image of $C_{10}$ in $X^{\prime}$ has intersection number with $K_{Y}$ which cannot be an integer, a contradiction. 
Lemma 2.2. $\operatorname{ord}(g) \neq 2.30,3.20,4.15,6.10$.

Proof. These cases are much simpler than the previous one, and are contained in [7], Lemmas 4.5 and 4.7 .

Lemma 2.3. If $\operatorname{ord}(g)=5.12$, then

$$
\left[g^{*}\right]=\left[1, \zeta_{12}: 4,1, \zeta_{60}: 16\right] .
$$

Proof. Since $g^{12}$ is symplectic of order 5 ,

$$
\left[g^{12 *}\right]=\left[1,1.5,\left(\zeta_{5}: 4\right) .4\right]
$$

and for any positive integer $a$ dividing $12, \operatorname{Fix}\left(g^{a}\right) \subset \operatorname{Fix}\left(g^{12}\right)$ and

$$
0 \leq e\left(g^{a}\right) \leq e\left(g^{12}\right)=4 .
$$

By Proposition 1.2, $\zeta_{12} \in\left[g^{*}\right]$. Thus we infer that

$$
\left[g^{*}\right]=\left[1, \zeta_{12}: 4, \pm 1, \eta_{1}, \ldots, \eta_{16}\right]
$$

where $\left[\eta_{1}, \ldots, \eta_{16}\right]$ is a combination of $\zeta_{5}: 4, \zeta_{10}: 4, \zeta_{15}: 8, \zeta_{20}: 8, \zeta_{30}: 8, \zeta_{60}: 16$ and the first eigenvalue corresponds to a $g$-invariant ample divisor.

Assume that $\left[\eta_{1}, \ldots, \eta_{16}\right]$ contains $\left[\zeta_{15}: 8\right]$ or $\left[\zeta_{30}: 8\right]$. Then

$$
\left[g^{2 *}\right]=\left[1,\left(\zeta_{6}: 2\right) .2,1, \zeta_{15}: 8, \tau_{1}, \ldots, \tau_{8}\right]
$$

where $\left[\tau_{1}, \ldots, \tau_{8}\right]$ is a combination of $\zeta_{5}: 4, \zeta_{10}: 4, \zeta_{15}: 8$, hence $\sum \tau_{j} \geq-2$ and $e\left(g^{2}\right)=\operatorname{Tr}\left(g^{2 *} \mid H^{*}(X)\right)=7+\sum \tau_{j} \geq 5$, contradicting $e\left(g^{2}\right) \leq 4$.

Assume that $\left[\eta_{1}, \ldots, \eta_{16}\right]$ contains $\left[\zeta_{20}: 8\right]$. In this case,

$$
\left[g^{2 *}\right]=\left[1,\left(\zeta_{6}: 2\right) .2,1,\left(\zeta_{10}: 4\right) .2, \tau_{1}, \ldots, \tau_{8}\right]
$$

where $\left[\tau_{1}, \ldots, \tau_{8}\right]$ is a combination of $\zeta_{5}: 4, \zeta_{10}: 4, \zeta_{15}: 8$. Since $\sum \tau_{j} \geq-2$, $e\left(g^{2}\right)=\operatorname{Tr}\left(g^{2 *} \mid H^{*}(X)\right)=8+\sum \tau_{j} \geq 6$, contradicting $e\left(g^{2}\right) \leq 4$.

Assume that $\left[\eta_{1}, \ldots, \eta_{16}\right]$ is a combination of $\zeta_{5}: 4, \zeta_{10}: 4$. Then

$$
\left[g^{6 *}\right]=\left[1,-1.4,1,\left(\zeta_{5}: 4\right) .4\right]
$$

and $e\left(g^{6}\right)=\operatorname{Tr}\left(g^{6 *} \mid H^{*}(X)\right)=-4$, contradicting $e\left(g^{6}\right) \geq 0$.

Therefore $\left[\eta_{1}, \ldots, \eta_{16}\right]=\left[\zeta_{60}: 16\right]$. Now Lemma 1.9 applies.

\section{Proof of Theorem 0.1.}

(1) follows from Lemmas 2.1 and 2.2 .

(2) We know ord $(g)=5.12$. By Lemma 2.3 we can apply Lemma 1.9, and will use the elliptic structure and the notation there. Let

$$
y^{2}+x^{3}+A\left(t_{0}, t_{1}\right) x+B\left(t_{0}, t_{1}\right)=0
$$

be the Weierstrass equation of the $g$-invariant elliptic pencil, where $A$ (resp. $B$ ) is a binary form of degree 8 (resp. 12). By Lemma 1.9, $g$ leaves invariant the section $R$ and the action of $g$ on the base of the fibration $\psi: X \rightarrow \mathbf{P}^{1}$ is of order 10. After a linear change of the coordinates $\left(t_{0}, t_{1}\right)$ we may assume that $g$ acts on the base by

$$
g:\left(t_{0}, t_{1}\right) \mapsto\left(t_{0}, \zeta_{60}^{6} t_{1}\right)
$$


for some primitive 60 th root of unity $\zeta_{60}$. Fix this $\zeta_{60}$. There are four primitive 60 th roots of unity whose 6 th powers equal to $\zeta_{60}^{6}$ :

$$
\zeta_{60}, \zeta_{60}^{11}, \zeta_{60}^{31}, \zeta_{60}^{41}
$$

We know that $g$ preserves two cuspidal fibres $F_{0}, F_{\infty}$ and makes the remaining 10 cuspidal fibres to form one orbit. Thus the discriminant polynomial

$$
\Delta=-4 A^{3}-27 B^{2}=c t_{0}^{2} t_{1}^{2}\left(t_{1}^{10}-t_{0}^{10}\right)^{2}
$$

for some constant $c \in k$, as it must have two double roots (corresponding to the fibres $\left.F_{0}, F_{\infty}\right)$ and one orbit of double roots. We know that the zeros of $A$ correspond to either cuspidal fibres or non-singular fibres with "complex multiplication" automorphism of order 6 . Since this set is invariant with respect to the order 10 action of $g$ on the base, we see that the only possibility is $A=0$. Then the above Weierstrass equation can be written in the form

$$
y^{2}+x^{3}+a t_{0} t_{1}\left(t_{1}^{10}-t_{0}^{10}\right)=0
$$

for some constant $a$. A suitable linear change of variables makes $a=1$ without changing the action of $g$ on the base. Thus

$$
X \cong X_{60}
$$

as an elliptic surface. Let $t=t_{1} / t_{0}$. Since $\operatorname{ord}(g)=5.12$,

$$
g^{*}\left(\frac{d x \wedge d t}{y}\right)=\mu \frac{d x \wedge d t}{y}
$$

for some primitive 12 th root of unity $\mu$. For the above fixed $\zeta_{60}$

$$
\mu=\zeta_{60}^{5}, \quad \zeta_{60}^{25}, \quad \zeta_{60}^{35} \text { or } \zeta_{60}^{55} \text {. }
$$

Note that $\left(\zeta_{60}^{11}\right)^{5}=\zeta_{60}^{55}, \quad\left(\zeta_{60}^{31}\right)^{5}=\zeta_{60}^{35}, \quad\left(\zeta_{60}^{41}\right)^{5}=\zeta_{60}^{25}$. Thus by replacing $\zeta_{60}$ by one of the four conjugates, one always has

$$
g^{*}(t)=\zeta_{60}^{6} t, g^{*}\left(\frac{d x \wedge d t}{y}\right)=\zeta_{60}^{5} \frac{d x \wedge d t}{y} .
$$

Since $g^{10}$ is of order 6 and acts trivially on the base, it is a complex multiplication of order 6 on a general fibre, so

$$
g^{10}\left(x, y, t_{0}, t_{1}\right)=\left(\zeta_{6}^{2} x, \zeta_{6}^{3} y, t_{0}, t_{1}\right) .
$$

Note that

$$
\operatorname{Fix}(g)=\left\{\text { the two cusps of } F_{0} \text { and } F_{\infty}\right\} \cup\left(R \cap F_{0}\right) \cup\left(R \cap F_{\infty}\right) .
$$

Analysing the local action of $g$ at the fixed point $\left(x, y, t_{0}, t_{1}\right)=(0,0,1,0)$, the cusp of $F_{0}$, we infer that

$$
g\left(x, y, t_{0}, t_{1}\right)=\left(\zeta_{60}^{2} x, \zeta_{60}^{3} y, t_{0}, \zeta_{60}^{6} t_{1}\right)
$$

Here we first determine the linear terms, then see that the higher degree terms must vanish. This completes the proof of Theorem 0.1 in the positive characteristic case. 


\section{Proof: the complex case}

Throughout this section, $X$ is a complex K3 surface.

A non-projective K3 surface cannot admit a non-symplectic automorphism of finite order (see $[10,16]$ ), and its automorphisms of finite order are symplectic, hence of order $\leq 8$. Thus we may assume that $X$ is projective. The proofs of Lemmas 1.6, 1.9, 2.1-2.3 go word for word, once the $l$-adic cohomology $H_{\text {et }}^{2}\left(X, \mathbb{Q}_{l}\right)$ is replaced by the integral singular cohomology $H^{2}(X, \mathbb{Z})$, and Proposition 1.4 by the usual topological Lefschetz formula. "Proof of Theorem 0.1" also goes word for word.

\section{Acknowledgments}

The author is grateful to the referee for careful reading. This research was partially completed while the author was visiting the Institute for Mathematical Sciences, National University of Singapore in July 2013. The research was supported by the National Research Foundation of Korea (NRF-2007-0093858).

\section{References}

[1] P. Deligne and G. Lusztig, Representations of reductive groups over finite fields, Ann. Math. (2) $\mathbf{1 0 3}(1)$ (1976), 103-161.

[2] I. Dolgachev and J. Keum, Finite groups of symplectic automorphisms of K3 surfaces in positive characteristic, Ann. Math. 169 (2009), 269-313

[3] _ K3 surfaces with a symplectic automorphism of order 11, J. Eur. Math. Soc. 11 (2009), 799-818

[4] Y. Goto, The Artin invariant of supersingular weighted Delsarte surfaces, J. Math. Kyoto Univ., 36 (1996), 359-363.

[5] D. Hwang and J. Keum, The maximum number of singular points on rational homology projective planes, J. Algebraic Geom. 20 (2011), 495-523.

[6] L. Illusie, Report on crystalline cohomology, in "Algebraic Geometry, Arcata 1974", Proc. Symp. Pure math. 29, AMS, pp. 459-478

[7] J. Keum, Orders of automorphisms of K3 surfaces, arXiv:1203.5616 [math.AG]

[8] J. Keum, K3 surfaces with an automorphism of order 66, the maximum possible, arXiv:14026803 [math. AG]

[9] N. Machida and K. Oguiso, On K3 surfaces admitting finite non-symplectic group actions, J. Math. Sci. Univ. Tokyo 5 (1998), 273-297

[10] V.V. Nikulin, Finite groups of automorphisms of Kählerian surfaces of type K3, Uspehi Mat. Nauk 31(2) (1976), Trans. Moscow Math. Soc., 38 (1980), 71-135

[11] K. Oguiso, A characterization of the Fermat quartic K3 surface by means of finite symmetries, Compos. Math. 141 (2005), 404-424

[12] A. Ogus, Supersingular K3 crystals, in 'Journées de Géometrie Algébrique de Rennes,' Asterisque, 64 (1979), 3-86

[13] _ A crystalline Torelli theorem for supersingular K3 surfaces, in 'Arithmetic and Geometry, Vol. II,' Progress in Mathematics, 36, Birkhäuser Boston, Boston, MA, 1983, 361394.

[14] T. Shioda, An explicit algorithm for computing the Picard number of certain algebraic surfaces, Amer. J. Math. 108 (1986), 415-432

[15] _ Supersingular K3 surfaces with big Artin invariant, J. Reine Angew. Math. 381 (1987), $205-210$

[16] K. Ueno, Classification theory of algebraic varieties and compact complex spaces, Springer Lecture Notes in Mathematics 439, 1975,

[17] G. Xiao, Non-symplectic involutions of a K3 surface, unpublished.

School of Mathematics, Korea Institute for Advanced Study, Seoul 130-722, Korea

E-mail address: jhkeum@kias.re.kr 